OPEN ACCESS

Edited by: Jorg Tost,

Commissariat à l'Energie Atomique et aux Energies Alternatives, France

Reviewed by:

Frederiqe Ponchel,

University of Leeds, United Kingdom

Marina V. Nemtsova,

I.M. Sechenov First Moscow State

Medical University, Russia

*Correspondence:

Shicheng Guo

Shicheng.Guo@wisc.edu;

shihcheng.guo@gmail.com

Dongyi He

hedongyi1967@shutcm.edu.cn

${ }^{t}$ These authors have contributed

equally to this work

Specialty section:

This article was submitted to Epigenomics and Epigenetics,

a section of the journal

Frontiers in Genetics

Received: 16 April 2020

Accepted: 06 July 2020

Published: 04 August 2020

Citation:

Guo S, Xu L, Chang C, Zhang R, Jin Y and He D (2020) Epigenetic

Regulation Mediated by Methylation in the Pathogenesis and Precision

Medicine of Rheumatoid Arthritis.

Front. Genet. 11:811.

doi: 10.3389/fgene.2020.00811

\section{Epigenetic Regulation Mediated by Methylation in the Pathogenesis and Precision Medicine of Rheumatoid Arthritis}

\author{
Shicheng Guo ${ }^{1,2 * t}$, Lingxia X $u^{3,44}$, Cen Chang ${ }^{3,44}$, Runrun Zhang ${ }^{3,4}$, Yehua Jin ${ }^{3,4}$ and \\ Dongyi $\mathrm{He}^{3,4 *}$
}

\begin{abstract}
${ }^{1}$ Department of Medical Genetics, School of Medicine and Public Health, University of Wisconsin-Madison, Madison, WI, United States, ${ }^{2}$ Center for Precision Medicine Research, Marshfield Clinic Research Institute, Marshfield, WI, United States, ${ }^{3}$ Department of Rheumatology, Guanghua Hospital, Shanghai University of Traditional Chinese Medicine, Shanghai, China, ${ }^{4}$ Institute of Arthritis Research in Integrative Medicine, Shanghai Academy of Traditional Chinese Medicine, Shanghai, China
\end{abstract}

Rheumatoid arthritis $(R A)$ is a complex disease triggered by the interaction between genetics and the environment, especially through the shared epitope (SE) and cell surface calreticulin (CSC) theory. However, the available evidence shows that genetic diversity and environmental exposure cannot explain all the clinical characteristics and heterogeneity of RA. In contrast, recent studies demonstrate that epigenetics play important roles in the pathogenesis of RA, especially DNA methylation and histone modification. DNA methylation and histone methylation are involved in innate and adaptive immune cell differentiation and migration, proliferation, apoptosis, and mesenchymal characteristics of fibroblast-like synoviocytes (FLS). Epigenetic-mediated regulation of immune-related genes and inflammation pathways explains the dynamic expression network of RA. In this review, we summarize the comprehensive evidence to show that methylation of DNA and histones is significantly involved in the pathogenesis of RA and could be applied as a promising biomarker in the disease progression and drug-response prediction. We also explain the advantages and challenges of the current epigenetics research in RA. In summary, epigenetic modules provide a possible interface through which genetic and environmental risk factors connect to contribute to the susceptibility and pathogenesis of RA. Additionally, epigenetic regulators provide promising drug targets to develop novel therapeutic drugs for RA. Finally, DNA methylation and histone modifications could be important features for providing a better RA subtype identification to accelerate personalized treatment and precision medicine.

\footnotetext{
Keywords: epigenetic, methylation, rheumatoid arthritis, pathogenesis, regulation
} 


\section{INTRODUCTION}

Rheumatoid arthritis (RA) is an autoimmune disease characterized by synovial hyperplasia and joint destruction (Lee et al., 2017). Its onset is progressive and invasive, which can lead to joint deformity and disability. In the past decades, linkage analysis and genome-wide association studies (GWAS) have identified $>100$ susceptibility genes, especially shared epitopes (SE), in HLA genes (Okada et al., 2019). Cell surface calreticulin (CSC), mediated by PTPN22 and PADI4, has successfully been used to explain the onset of RA (Okada et al., 2014). However, the pathogenesis of RA is still not fully understood. Furthermore, the majority of the current RA drugs have not been developed against GWAS targets but cytokine and inflammatory pathways. RA-associated genetics thoroughly explain neither the heterogeneity of the clinical characteristics nor the treatment differences among patients with RA. In recent years, more and more studies have started to focus on the role of epigenetics in RA (Ai et al., 2018; Guo et al., 2019, 2020; Tseng et al., 2019a) and investigate the contribution of epigenetics to the heterogeneity of RA. DNA methylation and histone modifications, important epigenetic modifications that affect the expression of immune-related genes and inflammation progression (Meng et al., 2015; Lawrence et al., 2016) have become promising mechanisms to explain the pathogenesis of RA (Mazzone et al., 2019). Numerous studies have found that methylation in immune cells may lead to RA progression through coordinated control of immune cell differentiation and function (Qiu et al., 2017; Meng et al., 2019). In this review, we systematically summarize the progress of methylation research (DNA and histone) to enhance the understanding of RA pathogenesis. We also summarize the pieces of evidence that show methylation as an interface to connect genetic and environmental exposures and as a promising biomarker for diagnosis, treatment, and subtype identification. Finally, we show that epigenetic modules are promising novel biomarkers and drug targets for the next-generation personalized treatment and precision medicine.

\section{GENOME-WIDE METHYLATION PROFILING TO IDENTIFY RA-ASSOCIATED EPIGENETIC VARIANTS}

DNA methylation is an important epigenetic modification that is involved in the regulation of gene expression and transcript splicing. Genome-wide identification of abnormal DNA methylation variations in FLS, innate and adopted immune cells, including $\mathrm{B}$ cells and $\mathrm{T}$ cells, provide a full spectrum of epigenetic pattern changes during the onset and progression of RA. Abnormal DNA methylation can be found at a very early stage of RA. Compared with normal synovial fibroblasts (SF), evidence shows that the $\mathrm{CpG}$ island located in the promoter region of PM20D1, EN1, and SHROOM1 are hypermethylated in very early RA-derived synovial fibroblasts (veRASF). MFAP2, RIMBP2, IRX6, DDR1, and HLA-C are found hypermethylated in established, long-standing RA-SF (estRASF). DNA methylation profiles in cadherin, integrin, and Wnt cell adhesion signaling pathways; actin cytoskeleton components; and antigen presentation pathways notably change in veRASF and estRASF (Karouzakis et al., 2018). Compared with early RA (ERA), the global DNA methylation level is lower in the cell migration, differentiation, and adhesion pathways in long-standing RA (LRA), which may contribute to cell proliferation, differentiation, migration, and transition to chronic RA (Ai et al., 2015). DNA methylation changes in these pathways show a similar pattern with human cancers, implicating that synovial hyperplasia and invasion may be a shared underlying mechanism with human cancer metastasis (Wang et al., 2015). Moreover, extracellular matrix (ECM), cholesterol biosynthesis, and immune system pathways are also significantly enriched in RA high-risk individuals, indicating that DNA methylation signals may be useful in early diagnosis or risk evaluation of RA (Karouzakis et al., 2019).

Genome-wide methylation change was also found in $\mathrm{T}$ and B lymphocytes (Glossop et al., 2013, 2014; Rhead et al., 2017; Guderud et al., 2020). A study identified 150 and 113 CpG loci with unique methylation characteristics in $\mathrm{T}$ and $\mathrm{B}$ lymphocytes in patients with ERA (Glossop et al., 2016). Evidence shows that ARSB and DUSP22 are hypermethylated and GALNT9 and MGMT are found hypomethylated in the T lymphocytes (Glossop et al., 2014). Interestingly, DUSP22 codes for a protein tyrosine phosphatase that negatively regulates STAT3 and IL-6/STAT3 signaling pathways, indicating DNA methylationmediated DUSP22 silencing, might be a fundamental effect to activate STAT signaling in RA (Sekine et al., 2006). BARX2, ASB1, ADAMTS17, and MGMT are found hypomethylated in the B lymphocytes and can be used to distinguish patients with RA from healthy individuals (Glossop et al., 2014).

Through genome-wide methylation analysis, the discovery of shared methylated regions and pathways in multiple immune diseases may suggest the existence of the same pathogenesis. A recent study identified 337 differential methylated genes shared between RA and Parkinson's Disease (PD), which provides new evidence for the shared biological mechanism between RA and PD (Tang et al., 2018). Another study found that mitochondrial L-carnitine shuttle and PTEN signaling pathways are simultaneously differentially expressed in RA, systemic sclerosis (SSc), and systemic lupus erythematosus (SLE) (Hudson et al., 2017). In other recent studies, genome-wide DNA methylation profiles revealed common epigenetic patterns of interferon-related genes in multiple autoimmune diseases, including Graves' disease (GD), RA, SLE, and SSc (Guo et al., 2017; Ding et al., 2018; Chen et al., 2019).

The methylation difference could also explain the different clinical manifestations and mechanisms in different autoimmune diseases. For example, compared with osteoarthritis (OA), 523 low-methylated regions are specific to RA. The regions overlap with specific motifs of transcription factors, such as GLI1, RUNX2, and TFAP2A/C, which promote the proliferation 
of synovial cells and the development and migration of plasmacytoid dendritic cells in RA (Ham et al., 2019). In contrast with OA, in which C18orf45, LMO4, MAP3K5, ODZ4, PKNOX2, SEPT11, MSRA, and MIR155HG are hypomethylated, PRDM16 is hypermethylated in RA (Glossop et al., 2015). CCR6, CMTM5, $I L-10 R, I L-21 R$, and $I L-32$ are found hypermethylated in SLE and primary Sjögren's syndrome (pSS), and they are also hypomethylated in RA (Wang et al., 2018). Here, we summarize all the differential methylated genes and construct an interaction network to show the relationship among these differential methylation genes in RA, using the annotations from ingenuity pathway analysis (IPA). We find that these differential methylated genes are not independent, but exhibit an interesting network (Figure 1 and Supplementary Table S1). These studies emphasize that changes in DNA methylation among different autoimmune diseases should be investigated in parallel to identify shared, disease-specific epigenetic dysfunctional elements.

\section{EPIGENETIC REGULATORY ROLES IN THE UNDERLYING PATHOGENESIS OF RHEUMATOID ARTHRITIS}

Rheumatoid arthritis fibroblast-like synoviocytes (RA-FLS) are involved in the release of inflammatory mediators and matrix-degrading enzymes, which are key effector cells leading to synovial inflammation and destruction of bone and cartilage (Neumann et al., 2010). The changes in DNA methylation in RA-FLS play important roles in the pathogenesis of RA. Hypomethylation in RA-FLS may be caused by the downregulation of DNMT1 and DNMT3A after inflammatory environmental stimulation (Nakano et al., 2013). Hypomethylation-mediated overexpression of TBX5 in RA-FLS increases the expression of IL-8, CXCL12, and CCL20, which enhances the inflammatory response in RA (Karouzakis et al., 2014). Hypermethylation of EBF3 and IRX1 in RA-FLS mediates the TNF- $\beta$ pathway and affects the proliferation, apoptosis, and mesenchymal characteristics of RA-FLS (Park et al., 2013). In addition, limb bud and heart development $(L B H)$ is a transcription regulator of the cell cycle and is involved in the control of cell growth and proliferation. The activity of $\mathrm{LBH}$ decreases by enhanced methylation, which may contribute to synovial hyperplasia by initiating the cell cycle (Ekwall et al., 2015; Hammaker et al., 2016).

The critical role of methylation changes in peripheral blood mononuclear cells (PBMCs) during the pathogenesis of RA was also extensively investigated. The demethylation levels in the promoter regions of IFNG and CNS-1 in peripheral blood CD4+ $\mathrm{T}$ cells of patients with RA increase, which establishes stable effector/memory during Th1-cell interaction (Dong et al., 2013). In addition, DNA methylation in the promoter region of CTLA-4 inhibits the activation of the tryptophan-degrading enzyme indoleamine 2,3-dioxygenase pathway, which results in the defunctionalization of Tregs (Cribbs et al., 2014). Further evidence shows that activation of $P R L R$ mediates the demethylation of TNF- $\alpha$ in peripheral CD14+ monocytes and increases the release of TNF- $\alpha$ (Tang et al., 2014). Hypomethylation of ZBTB38 decreases IL1R2 expression in $B$ cells to interfere with the anti-inflammatory pathway (Ocsko et al., 2018). Hypermethylation of the AHR promoter region is associated with the formation of germinal center in the B cells (Toth et al., 2019). Evidence shows that demethylation in the proximal promoter region of $E R$ mediates its increased expression in peripheral blood lymphocytes of RA patients, which is found to induce the occurrence of RA (Liu et al., 2014). Hypomethylation of DNA in apoptotic $\mathrm{CD} 4+\mathrm{T}$ cells upregulates the production of IL-6 in macrophages and downregulates the production of TGF- $\beta$ in the DCs and B cells (Notley et al., 2017). This evidence demonstrated that DNA methylation plays important roles in the pathogenesis of RA.

\section{DNA METHYLATION AS A BIOMARKER FOR RHEUMATOID ARTHRITIS DIAGNOSIS}

At present, the serological markers used for the diagnosis of RA are anti-citrullinated protein antibodies (ACPAs) and rheumatoid factor (RF). However, the sensitivity of the test is only about $\sim 70 \%$ (Nishimura et al., 2007). It is urgent to find novel markers to increase the diagnosis accuracy, disease onset prediction, and disease progression. Methylation levels of SHROOM1 in ERA are substantially increased, which can be applied as an early diagnosis biomarker (Karouzakis et al., 2018). Also, the MHC region is found hypomethylated in both PBMCs and the whole blood (van Steenbergen et al., 2014). Furthermore, CD86, RAB20, XAF1, FOLR3, LTBR, KCNH8, DOK7, PDGFA, PITPNM2, and CELSR1 in B cells show significant methylation differences in RA (Tseng et al., 2019b). In another study, S100A6 and $E F C A B 4 B$ promoter regions in the whole blood and IFNrelated genes (IFIT1, IRF7, MX1, OAS1, USP18, RSAD2, IFI44L) in CD4+ $\mathrm{T}$ cells are hypomethylated, indicating these methylation signals might be used as biomarkers for RA diagnosis (Svendsen et al., 2016; Chen et al., 2019). $A Z U 1, L T B R$, and RTEL1 are hypomethylated and involved in the autoimmune signaling cascade, indicating potential roles as epigenetic susceptibility markers (Wang et al., 2018). Hypomethylation of CYP2E1 is associated with disease activity and can be used as a disease activity marker (Mok et al., 2018). CD1C, TNFSF10, C6ORF10, and UBASH3A have the potential to be used as RA risk markers (Julia et al., 2017; Anaparti et al., 2019; Guderud et al., 2020). Finally, except gene-based methylation biomarkers, some other pathway or genome-wide indicators also show interesting biomarker performances. For example, a recent study showed that methylation-derived neutrophil-to-lymphocyte ratio (mNLR), detected from peripheral blood DNA, is increased during RA onset (Ambatipudi et al., 2018). Overall, all these pieces of evidence demonstrate that DNA methylation could be used as a robust biomarker for disease 


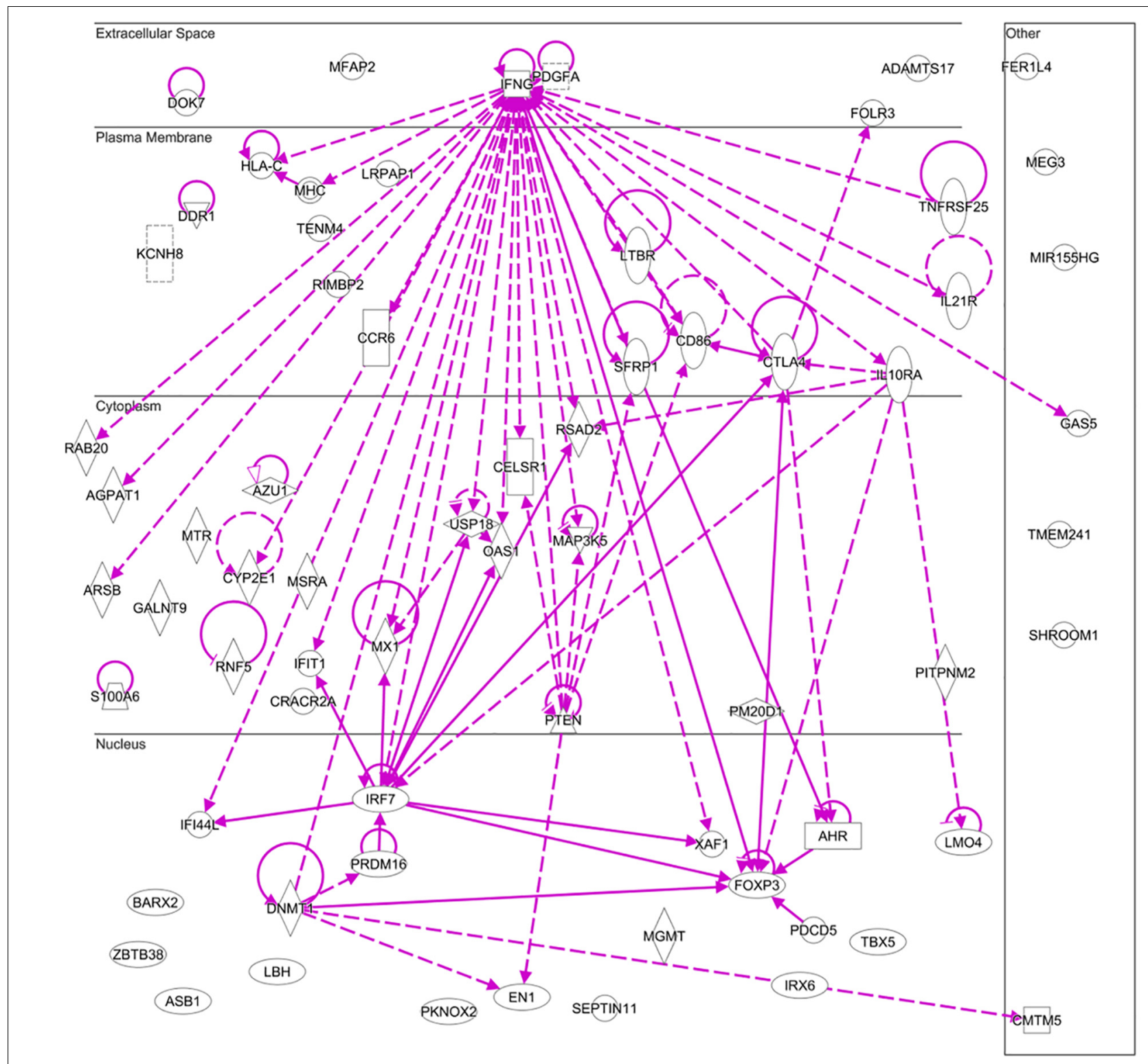

FIGURE 1 | Interaction network constructed by including all differential methylation genes reported in rheumatoid arthritis using an ingenuity pathway analysis (IPA). In this figure, we applied a grow algorithm on the IPA database to connect all the differentially methylated genes with direct and indirect links according to ingenuity core pathway. Gene products were arranged graphically based on the location/function of the associated proteins. Shapes and lines with different styles do not have special representation, but they make different gene productions easy to recognize.

risk assessment and progression, and we expect more future advancements in this field.

\section{EPIGENETIC MODULES MEDIATED BY METHYLATION ARE PROMISING RA DRUG TARGETS}

Epigenetic modifications are dynamically variable, and their reversibility is an attractive characteristic to develop new drugs. RA is highly related to the functional defects of Tregs, and FOXP3 stabilizes the immune regulatory function of Tregs. A recent study showed that daurinol induces hypomethylation of FOXP3, promotes Tregs differentiation and stabilization, inhibits Th17 differentiation through the Nrp1-Pten-Akt-Foxp3 signaling pathway, and alleviates the severity of RA (Park et al., 2019). In another study, expansion of Tregs in vitro with rapamycin was shown to improve the function and stability of Tregs, which maintain FOXP3 high expression and strong inhibitory ability, in which TNFR2 maintains FOXP3 expression by restricting DNA methylation (Rossetti et al., 2015). These pieces of evidence indicate hypomethylation of FOXP3, which may present an interesting drug target for the treatment of RA. 
Meanwhile, evidence shows that methotrexate (MTX) can restore Treg inhibition through the demethylation of FOXP3 upstream enhancer and increases the expression of FOXP3 and CTLA-4, providing a new mechanism for MTX (Cribbs et al., 2014). MTX can also reverse DNA hypomethylation in $\mathrm{T}$ cells, B cells, and monocytes in patients with RA (de Andres et al., 2015). Furthermore, patients with higher baseline global DNA methylation levels in RA exhibit lower MTX response (Gosselt et al., 2019). MTX can also reduce MTR expression in rheumatoid nodules, affecting remethylation mediated by $M T R$ and $M T R R$, indicating the important roles of DNA methylation in RA pathogenesis and drug response mechanism (Houlder et al., 2017). Recently, methylation inhibitors (5-Azadc) showed the ability to upregulate PTEN; downregulate HOTTIP; weaken the enrichment of $D N M T 3 B$ in the SFRP1 promoter region; and downregulate the expression of $\beta$-catenin, TNF-a, IL-6, IL-1 $\beta$, and CCL2 (Miao et al., 2013; Li X.F. et al., 2019; Hu et al., 2020). These changes further regulate the AKT and Wnt signaling pathways to aid the remission of RA. SFRP4 is a negative regulator of the Wnt signaling pathway. Evidence shows that $m i R-152$ indirectly upregulates SFRP4 by decreasing the expression of DNMT1 and reduces FLS proliferation (Miao et al., 2014).

The immunomodulatory role of etanercept and adalimumab in mononuclear cells is demonstrated by downregulating the expression of methyltransferase and trimethylation of $\mathrm{H} 3 \mathrm{~K} 4$, $\mathrm{H} 3 \mathrm{~K} 27$, H3K36, and H3K79 at the CCL2 promoter (Lin et al., 2017). Daphnetin reduces the expression of DNMT1, DNMT3A, and DNMT3B in collagen-induced arthritis rat synovial cells, leading to demethylation of pro-apoptotic genes DR3, PDCD5, FasL, and $p 53$ and increasing the expression of pro-apoptotic genes (Shu et al., 2014). The combination of SSAT1 inhibitor DA and methyl donor S-adenosyl methionine can significantly improve overall DNA hypomethylation status in RA-FLS and reduce the adhesion of RA-FLS (Neidhart et al., 2014). Another interesting study shows hypermethylation of the promoters FER1L4, GAS5, and MEG3, leading to their downregulation, which promotes the release of proinflammatory factors, contributing to the pathogenesis of RA. Therefore, hypomethylation recovery of FER1L4, GAS5, and MEG3 may be a potential therapeutic target for RA (Liu et al., 2019; Yu et al., 2019; Li et al., 2020). Finally, DNA methylation of LBH, CASP8, OLIG3, IRF5, HLA-G, ELMO1, TRHDE, SLCO1C1, PLD4, AIRE, and HLA-DQA1 is involved in the pathogenesis of RA and is recommended as a promising novel therapeutic target (Fan et al., 2016).

In addition, methylation also provides novel biomarkers to predict drug responses. There are four CpGs within exon seven of lipoprotein receptor-associated protein 1 (LRPAP1) that are more methylated in non-responders than in good responders, which can be used as a response marker of etanercept (TNF-alpha inhibitor) treatment (Plant et al., 2016). Disease-modifying antirheumatic drug (DMARD) reverses the hypomethylation of RNF5 and AGPAT1 promoter regions induced by smoking in patients with RA; therefore, it could be considered as a therapeutic target for RA (Svendsen et al., 2016). Meanwhile, two methylation loci (cg03018489 and cg14345882) are significantly correlated with DMARD treatment response (Glossop et al., 2017). In the good responders to MTX, methylation levels of cg23700278, cg27427581, cg04334751, and cg26764200 increase significantly after MTX treatment (Nair et al., 2019). In summary, the drugs that improve the abnormal methylation could be promising for the treatment of RA, and the genes involved in the epigenetic regulation of RA could be considered as novel drug targets for pharmaceutical companies and to explain the novel mechanism of drug action. Meanwhile, the differential DNA methylation can be used as a promising biomarker to predict the drug responses.

\section{THE ADVANCEMENT OF HISTONE METHYLATION RESEARCH IN RHEUMATOID ARTHRITIS}

Histone methylation is also involved in the pathogenesis of RA. Histone $\mathrm{H} 3$ trimethylated at lysine 4 (H3K4me3) in SF is associated with the opening of arthritis-activated chromatin, making the promoters of pathogenic genes highly active to drive transcription (Ntougkos et al., 2017). A previous study shows that $\mathrm{H} 3 \mathrm{~K} 4 \mathrm{me} 3$ is increased in the promoter region of $M M P$, which is positively correlated with the expression of MMP-1, MMP-3, MMP-9, and MMP-13 in RA-FLS (Araki et al., 2016). Peptidylarginine deiminase type-4 (PADI4) inhibits p21 transcription by modifying histone $\mathrm{H} 3$ arginine at the p21 promoter region, which protects FLS from apoptosis and promotes the pathogenesis of RA (Fan et al., 2017). The expression of Jumonji domain-containing protein 3 (JMJD3) in RA-FLS is upregulated. JMJD3 specifically demethylates trimethylated lysine, which is directly involved in the activation of TLR2 through the demethylation of $\mathrm{H} 3 \mathrm{~K} 27 \mathrm{me} 3$ promoter and promotes RA inflammation (Wu et al., 2019). Aiming at the pathogenesis of RA caused by abnormal histone methylation is useful in improving the inflammation in RA. For example, the application of JMJD3 inhibitor, GSK-J4, inhibits the methylation of $\mathrm{H} 3 \mathrm{~K} 27 \mathrm{me} 3$ at the TLR2 promoter, significantly relieving the destruction and inflammation of articular cartilage ( Wu et al., 2019). Therefore, histone modification regulators are potential and promising drug targets for RA therapy and drug development.

\section{CHALLENGES AND OPPORTUNITIES OF THE CURRENT EPIGENETICS RESEARCH IN RHEUMATOID ARTHRITIS}

Research on epigenetics highlights the role of methylation changes in the pathogenesis, diagnosis, treatment, and prognosis of RA. DNA and histone methylation, including TBX5 (Karouzakis et al., 2014), FOXP3 (Kennedy et al., 2014), AHR (Toth et al., 2019), HKMT (Araki et al., 2018), and H3K4me3 (Araki et al., 2016), affect the proliferation, migration, apoptosis, and inflammation of immune cells, which explains 
the pathogenesis of RA. Preclinical differential methylation changes, such as the mNLR, contribute to early diagnosis of RA (Ambatipudi et al., 2018). The identified differential methylation genes can be applied as useful biomarkers to predict RA progression and disease severity and provide potential therapeutic targets for RA. Epigenetic modifications as drug targets could provide a new direction of pharmacological research for the development of novel drugs that alleviate clinical pressures of high toxicity, low efficiency, and high cost of the current medicine. For example, demethylation of FOXP3 is used as a biomarker to evaluate the therapeutic drug response, which provides a direction for the precision treatment of RA (Tabares et al., 2018). MALAT1 promotes CTNNB1 promoter methylation and inhibits the Wnt signaling pathway, and has been shown as an interesting potential therapeutic target for RA (Li G.Q. et al., 2019). In addition, DNA and histone methylation in individuals may change the response to drugs; identifying the drug response markers may be helpful for personalized medication development.

Although DNA methylation and histone methylation research have shown significant progress in RA, the current research presents several severe concerns. For example, there is no comprehensive study to investigate the relationship between differential methylation and disease severity and inflammation indicators. Future research should also strive to extended cohort studies to improve statistical robustness. Studies of differential methylation and pathways have examined the corresponding sites, but few functional tests have been performed. Different experimental approaches should be integrated to verify the results and obtain a solid conclusion with a large sample size. Moreover, further studies are needed to reveal the functions and targets of differential methylation and illuminate RA pathogenesis and drug discovery. We expect multi-omics analyses to be conducted to illustrate the roles of the interactions between epigenetic elements ( $5 \mathrm{mC}$ and histone modification) in multiple autoimmune diseases as well as the interaction between genetics and epigenetics for a better understanding of RA pathogenesis.

\section{REFERENCES}

Ai, R., Whitaker, J. W., Boyle, D. L., Tak, P. P., Gerlag, D. M., Wang, W., et al. (2015). DNA methylome signature in synoviocytes from patients with early rheumatoid arthritis compared to synoviocytes from patients with longstanding rheumatoid arthritis. Arthritis Rheumatol. 67, 1978-1980. doi: 10.1002/art. 39123

Ai, R. Z., Laragione, T., Hammaker, D., Boyle, D. L., Wildberg, A., Maeshima, K., et al. (2018). Comprehensive epigenetic landscape of rheumatoid arthritis fibroblast-like synoviocytes. Nat. Commun. 9:1921. doi: 10.1038/S41467-01804310-9

Ambatipudi, S., Sharp, G. C., Clarke, S. L. N., Plant, D., Tobias, J. H., Evans, D. M., et al. (2018). Assessing the role of DNA methylation-derived neutrophilto-lymphocyte ratio in rheumatoid arthritis. J. Immunol. Res. 2018:2624981. doi: $10.1155 / 2018 / 2624981$

Anaparti, V., Agarwal, P., Smolik, I., Mookherjee, N., and Elgabalawy, H. (2019). Whole blood targeted bisulfite sequencing validates differential methylation in C6ORF10 gene of patients with rheumatoid arthritis. J. Rheumatol. doi: 10.3899/jrheum.190376 [Epub ahead of print].

Araki, Y., Aizaki, Y., Sato, K., Oda, H., Kurokawa, R., and Mimura, T. (2018). Altered gene expression profiles of histone lysine methyltransferases and

\section{AUTHOR CONTRIBUTIONS}

SG and DH conceived of content. SG, LX, and CC drafted the review, which was edited by RZ and YJ. All authors contributed to the article and approved the submitted version.

\section{FUNDING}

This work was funded by the National Natural Science Foundation of China (81774114), Shanghai Chinese Medicine Development Office, Shanghai Chinese and Western Medicine Clinical Pilot Project (ZY(2018-2020)-FWTX-1010), Shanghai Chinese Medicine Development Office, Shanghai Traditional Chinese Medicine Specialty Alliance Project (ZY(2018-2020)FWTX-4017), and National Administration of Traditional Chinese Medicine, Regional Chinese Medicine (Specialist) Diagnosis and Treatment Center Construction ProjectRheumatology.

\section{ACKNOWLEDGMENTS}

We appreciate the IPA analysis support from the Key Laboratory of Genomic and Precision Medicine, Beijing Institute of Genomics, Chinese Academy of Sciences. The manuscript has previously appeared online, doi: 10.20944/preprints202004. 0237.v1.

\section{SUPPLEMENTARY MATERIAL}

The Supplementary Material for this article can be found online at: https://www.frontiersin.org/articles/10.3389/fgene. 2020.00811/full\#supplementary-material

TABLE S1 | Interaction network among rheumatoid arthritis differential methylation genes.

demethylases in rheumatoid arthritis synovial fibroblasts. Clin. Exp. Rheumatol. 36, 314-316.

Araki, Y., Tsuzuki Wada, T., Aizaki, Y., Sato, K., Yokota, K., Fujimoto, K., et al. (2016). Histone methylation and STAT-3 differentially regulate interleukin6-induced matrix metalloproteinase gene activation in rheumatoid arthritis synovial fibroblasts. Arthritis Rheumatol. 68, 1111-1123. doi: 10.1002/art. 39563

Chen, S., Pu, W., Guo, S., Jin, L., He, D., and Wang, J. (2019). Genome-wide DNA methylation profiles reveal common epigenetic patterns of interferonrelated genes in multiple autoimmune diseases. Front. Genet. 10:223. doi: 10. 3389/fgene.2019.00223

Cribbs, A. P., Kennedy, A., Penn, H., Read, J. E., Amjadi, P., Green, P., et al. (2014). Treg cell function in rheumatoid arthritis is compromised by ctla- 4 promoter methylation resulting in a failure to activate the indoleamine 2,3dioxygenase pathway. Arthritis Rheumatol. 66, 2344-2354. doi: 10.1002/art. 38715

de Andres, M. C., Perez-Pampin, E., Calaza, M., Santaclara, F. J., Ortea, I., Gomez-Reino, J. J., et al. (2015). Assessment of global DNA methylation in peripheral blood cell subpopulations of early rheumatoid arthritis before and after methotrexate. Arthritis Res. Ther. 17, 233. doi: 10.1186/s13075-0150748-5 
Ding, W., Pu, W., Wang, L., Jiang, S., Zhou, X., Tu, W., et al. (2018). Genomewide DNA methylation analysis in systemic sclerosis reveals hypomethylation of IFN-associated genes in CD4(+) and CD8(+) T cells. J. Invest. Dermatol. 138, 1069-1077. doi: 10.1016/j.jid.2017.12.003

Dong, J., Chang, H. D., Ivascu, C., Qian, Y., Rezai, S., Okhrimenko, A., et al. (2013). Loss of methylation at the IFNG promoter and CNS-1 is associated with the development of functional IFN-gamma memory in human CD4(+) T lymphocytes. Eur. J. Immunol. 43, 793-804. doi: 10.1002/eji.20124 2858

Ekwall, A. K., Whitaker, J. W., Hammaker, D., Bugbee, W. D., Wang, W., and Firestein, G. S. (2015). The rheumatoid arthritis risk gene $\mathrm{LBH}$ regulates growth in fibroblast-like synoviocytes. Arthritis Rheumatol. 67, 1193-1202. doi: 10. 1002/art.39060

Fan, L., Zong, M., Gong, R., He, D., Li, N., Sun, L. S., et al. (2017). PADI4 epigenetically suppresses p21 transcription and inhibits cell apoptosis in fibroblast-like synoviocytes from rheumatoid arthritis patients. Int. J. Biol. Sci. 13, 358-366. doi: 10.7150/ijbs. 16879

Fan, S., Li, C., Ai, R., Wang, M., Firestein, G. S., and Wang, W. (2016). Computationally expanding infinium HumanMethylation450 BeadChip array data to reveal distinct DNA methylation patterns of rheumatoid arthritis. Bioinformatics 32, 1773-1778. doi: 10.1093/bioinformatics/ btw089

Glossop, J. R., Emes, R. D., Nixon, N. B., Haworth, K. E., Packham, J. C., Dawes, P. T., et al. (2014). Genome-wide DNA methylation profiling in rheumatoid arthritis identifies disease-associated methylation changes that are distinct to individual T- and B-lymphocyte populations. Epigenetics 9, 1228-1237. doi: 10.4161/epi.29718

Glossop, J. R., Emes, R. D., Nixon, N. B., Packham, J. C., Fryer, A. A., Mattey, D. L., et al. (2016). Genome-wide profiling in treatment-naive early rheumatoid arthritis reveals DNA methylome changes in T and B lymphocytes. Epigenomics 8, 209-224. doi: 10.2217/epi.15.103

Glossop, J. R., Haworth, K. E., Emes, R. D., Nixon, N. B., Packham, J. C., Dawes, P. T., et al. (2015). DNA methylation profiling of synovial fluid FLS in rheumatoid arthritis reveals changes common with tissue-derived FLS. Epigenomics 7, 539-551. doi: 10.2217/epi. 15.15

Glossop, J. R., Nixon, N. B., Emes, R. D., Haworth, K. E., Packham, J. C., Dawes, P. T., et al. (2013). Epigenome-wide profiling identifies significant differences in DNA methylation between matched-pairs of T- and B-lymphocytes from healthy individuals. Epigenetics 8, 1188-1197. doi: 10.4161/epi. 26265

Glossop, J. R., Nixon, N. B., Emes, R. D., Sim, J., Packham, J. C., Mattey, D. L., et al. (2017). DNA methylation at diagnosis is associated with response to disease-modifying drugs in early rheumatoid arthritis. Epigenomics 9, 419-428. doi: 10.2217/epi-2016-0042

Gosselt, H. R., van Zelst, B. D., de Rotte, M., Hazes, J. M. W., de Jonge, R., and Heil, S. G. (2019). Higher baseline global leukocyte DNA methylation is associated with MTX non-response in early RA patients. Arthritis Res. Ther. 21:157. doi: 10.1186/s13075-019-1936-5

Guderud, K., Sunde, L. H., Flam, S. T., Maehlen, M. T., Mjaavatten, M. D., Lillegraven, S., et al. (2020). Rheumatoid arthritis patients, both newly diagnosed and methotrexate treated, show more DNA methylation differences in CD4(+) memory than in CD4(+) naive T cells. Front. Immunol. 11:194. doi: 10.3389/fimmu.2020.00194

Guo, S., Chang, C., Xu, L., Zhang, R., Jin, Y., Xiong, M., et al. (2020). Epigenetic regulation mediated by miRNA in the susceptibility and pathogenesis of rheumatoid arthritis. Preprints 2020:2020040241.

Guo, S., Liu, J., Jiang, T., Lee, D., Wang, R., Zhou, X., et al. (2019). (5R)-5-Hydroxytriptolide (LLDT-8) induces substantial epigenetic mediated immune response network changes in fibroblast-like synoviocytes from rheumatoid arthritis patients. Sci. Rep. 9:11155. doi: 10.1038/s41598-019-47 411-1

Guo, S., Zhu, Q., Jiang, T., Wang, R., Shen, Y., Zhu, X., et al. (2017). Genome-wide DNA methylation patterns in CD4+ T cells from Chinese Han patients with rheumatoid arthritis. Mod. Rheumatol. 27, 441-447. doi: 10.1080/14397595. 2016.1218595
Ham, S., Bae, J. B., Lee, S., Kim, B. J., Han, B. G., Kwok, S. K., et al. (2019). Epigenetic analysis in rheumatoid arthritis synoviocytes. Exp. Mol. Med. 51, 1-13. doi: 10.1038/s12276-019-0215-5

Hammaker, D., Whitaker, J. W., Maeshima, K., Boyle, D. L., Ekwall, A. H., Wang, W., et al. (2016). LBH gene transcription regulation by the interplay of an enhancer risk allele and DNA methylation in rheumatoid arthritis. Arthritis Rheumatol. 68, 2637-2645. doi: 10.1002/art. 39746

Houlder, E. L., Millier, M. J., Highton, J., Gwynne-Jones, D., Stamp, L. K., and Hessian, P. A. (2017). Expression of the genes facilitating methotrexate action within subcutaneous rheumatoid nodules. Clin. Exp. Rheumatol. 35, 943-947.

Hu, X., Tang, J., Hu, X., Bao, P., Deng, W., Wu, J., et al. (2020). Silencing of Long Non-coding RNA HOTTIP Reduces Inflammation in Rheumatoid Arthritis by Demethylation of SFRP1. Mol. Ther. Nucleic Acids 19, 468-481. doi: 10.1016/j. omtn.2019.11.015

Hudson, M., Bernatsky, S., Colmegna, I., Lora, M., Pastinen, T., Klein Oros, K., et al. (2017). Novel insights into systemic autoimmune rheumatic diseases using shared molecular signatures and an integrative analysis. Epigenetics 12, 433-440. doi: 10.1080/15592294.2017.130 3581

Julia, A., Absher, D., Lopez-Lasanta, M., Palau, N., Pluma, A., Waite Jones, L., et al. (2017). Epigenome-wide association study of rheumatoid arthritis identifies differentially methylated loci in B cells. Hum. Mol. Genet. 26, 2803-2811. doi: $10.1093 / \mathrm{hmg} / \mathrm{ddx} 177$

Karouzakis, E., Hahnlein, J., Grasso, C., Semmelink, J. F., Tak, P. P., Gerlag, D. M., et al. (2019). Molecular characterization of human lymph node stromal cells during the earliest phases of rheumatoid arthritis. Front. Immunol. 10:1863. doi: 10.3389/fimmu.2019.01863

Karouzakis, E., Raza, K., Kolling, C., Buckley, C. D., Gay, S., Filer, A., et al. (2018). Analysis of early changes in DNA methylation in synovial fibroblasts of RA patients before diagnosis. Sci. Rep. 8:7370. doi: 10.1038/s41598-018-24 240-2

Karouzakis, E., Trenkmann, M., Gay, R. E., Michel, B. A., Gay, S., and Neidhart, M. (2014). Epigenome analysis reveals TBX5 as a novel transcription factor involved in the activation of rheumatoid arthritis synovial fibroblasts. J. Immunol. 193, 4945-4951. doi: 10.4049/jimmunol.140 0066

Kennedy, A., Schmidt, E. M., Cribbs, A. P., Penn, H., Amjadi, P., Syed, K., et al. (2014). A novel upstream enhancer of FOXP3, sensitive to methylationinduced silencing, exhibits dysregulated methylation in rheumatoid arthritis Treg cells. Eur. J. Immunol. 44, 2968-2978. doi: 10.1002/eji.20144 4453

Lawrence, M., Daujat, S., and Schneider, R. (2016). Lateral thinking: how histone modifications regulate gene expression. Trends Genet. 32, 42-56. doi: 10.1016/ j.tig.2015.10.007

Lee, K. A., Min, S. H., Kim, T. H., Lee, S. H., and Kim, H. R. (2017). Magnetic resonance imaging-assessed synovial and bone changes in hand and wrist joints of rheumatoid arthritis patients. Ann. Rheum. Dis. 76, 1166-1166. doi: 10.1136/ annrheumdis-2017-eular.1507

Li, G. Q., Fang, Y. X., Liu, Y., Meng, F. R., Wu, X., Zhang, C. W., et al. (2019). MALAT1-driven inhibition of wnt signal impedes proliferation and inflammation in fibroblast-like synoviocytes through CTNNB1 promoter methylation in rheumatoid arthritis. Hum. Gene Ther. 30, 1008-1022. doi: 10.1089/hum.2018.212

Li, M., Wang, N., Shen, Z., and Yan, J. (2020). Long non-coding RNA growth arrest-specific transcript 5 regulates rheumatoid arthritis by targeting homeodomain-interacting protein kinase 2. Clin. Exp. Rheumatol. [Epub ahead of print].

Li, X. F., Chen, X., Bao, J., Xu, L., Zhang, L., Huang, C., et al. (2019). PTEN negatively regulates the expression of pro-inflammatory cytokines and chemokines of fibroblast-like synoviocytes in adjuvant-induced arthritis. Artif. Cells Nanomed. Biotechnol. 47, 3687-3696. doi: 10.1080/21691401.2019. 1661849

Lin, Y. C., Lin, Y. C., Huang, M. Y., Kuo, P. L., Wu, C. C., Lee, M. S., et al. (2017). Tumor necrosis factor-alpha inhibitors suppress CCL2 chemokine in 
monocytes via epigenetic modification. Mol. Immunol. 83, 82-91. doi: 10.1016/ j.molimm.2017.01.009

Liu, H. W., Lin, H. L., Yen, J. H., Tsai, W. C., Chiou, S. S., Chang, J. G., et al. (2014). Demethylation within the proximal promoter region of human estrogen receptor alpha gene correlates with its enhanced expression: implications for female bias in lupus. Mol. Immunol. 61, 28-37. doi: 10.1016/j.molimm.2014.05. 002

Liu, Y. R., Yang, L., Xu, Q. Q., Lu, X. Y., Ma, T. T., Huang, C., et al. (2019). Long noncoding RNA MEG3 regulates rheumatoid arthritis by targeting NLRC5. J. Cell. Physiol. 234, 14270-14284. doi: 10.1002/jcp.28 126

Mazzone, R., Zwergel, C., Artico, M., Taurone, S., Ralli, M., Greco, A., et al. (2019). The emerging role of epigenetics in human autoimmune disorders. Clin. Epigenet. 11:34. doi: 10.1186/s13148-019-0632-2

Meng, H., Cao, Y., Qin, J. Z., Song, X. Y., Zhang, Q., Shi, Y., et al. (2015). DNA methylation, its mediators and genome integrity. Int. J. Biol. Sci. 11, 604-617. doi: 10.7150/ijbs.11218

Meng, M., Liu, H. F., Chen, S. D., Zhao, H. J., Gao, X., Zhang, J. N., et al. (2019). Methylation of H3K27 and H3K4 in key gene promoter regions of thymus in RA mice is involved in the abnormal development and differentiation of iNKT cells. Immunogenetics 71, 489-499. doi: 10.1007/s00251-019-01 124-x

Miao, C. G., Huang, C., Huang, Y., Yang, Y. Y., He, X., Zhang, L., et al. (2013). MeCP2 modulates the canonical Wnt pathway activation by targeting SFRP4 in rheumatoid arthritis fibroblast-like synoviocytes in rats. Cell Signal 25, 598-608. doi: 10.1016/j.cellsig.2012.11.023

Miao, C. G., Yang, Y. Y., He, X., Huang, C., Huang, Y., Qin, D., et al. (2014). MicroRNA-152 modulates the canonical Wnt pathway activation by targeting DNA methyltransferase 1 in arthritic rat model. Biochimie 106, 149-156. doi: 10.1016/j.biochi.2014.08.016

Mok, A., Rhead, B., Holingue, C., Shao, X., Quach, H. L., Quach, D., et al. (2018). Hypomethylation of CYP2E1 and DUSP22 promoters associated with disease activity and erosive disease among rheumatoid arthritis patients. Arthritis Rheumatol. 70, 528-536. doi: 10.1002/art.40408

Nair, N., Plant, D., Verstappen, S. M., Isaacs, J. D., Morgan, A. W., Hyrich, K. L., et al. (2019). Differential DNA methylation correlates with response to methotrexate in rheumatoid arthritis. Rheumatology 59, 1364-1371. doi: 10. 1093/rheumatology/kez411

Nakano, K., Boyle, D. L., and Firestein, G. S. (2013). Regulation of DNA methylation in rheumatoid arthritis synoviocytes. J. Immunol. 190, 1297-1303. doi: 10.4049/jimmunol.1202572

Neidhart, M., Karouzakis, E., Jungel, A., Gay, R. E., and Gay, S. (2014). Inhibition of spermidine/spermine N1-acetyltransferase activity: a new therapeutic concept in rheumatoid arthritis. Arthritis Rheumatol. 66, 1723-1733. doi: 10.1002/art. 38574

Neumann, E., Lefevre, S., Zimmermann, B., Gay, S., and Muller-Ladner, U. (2010). Rheumatoid arthritis progression mediated by activated synovial fibroblasts. Trends Mol. Med. 16, 458-468. doi: 10.1016/j.molmed.2010. 07.004

Nishimura, K., Sugiyama, D., Kogata, Y., Tsuji, G., Nakazawa, T., Kawano, S., et al. (2007). Meta-analysis: diagnostic accuracy of anti-cyclic citrullinated peptide antibody and rheumatoid factor for rheumatoid arthritis. Ann. Intern. Med. 146, 797-808. doi: 10.7326/0003-4819-146-11-20070605000008

Notley, C. A., Jordan, C. K., McGovern, J. L., Brown, M. A., and Ehrenstein, M. R. (2017). DNA methylation governs the dynamic regulation of inflammation by apoptotic cells during efferocytosis. Sci. Rep. 7:42204. doi: 10.1038/srep 42204

Ntougkos, E., Chouvardas, P., Roumelioti, F., Ospelt, C., Frank-Bertoncelj, M., Filer, A., et al. (2017). Genomic responses of mouse synovial fibroblasts during tumor necrosis factor-driven arthritogenesis greatly mimic those in human rheumatoid arthritis. Arthritis Rheumatol. 69, 1588-1600. doi: 10.1002/art. 40128

Ocsko, T., Toth, D. M., Hoffmann, G., Tubak, V., Glant, T. T., and Rauch, T. A. (2018). Transcription factor Zbtb38 downregulates the expression of anti-inflammatory IL1r2 in mouse model of rheumatoid arthritis. Biochim. Biophys. Acta Gene Regul. Mech. 1861, 1040-1047. doi: 10.1016/j.bbagrm.2018. 09.007
Okada, Y., Eyre, S., Suzuki, A., Kochi, Y., and Yamamoto, K. (2019). Genetics of rheumatoid arthritis: 2018 status. Ann. Rheum. Dis. 78, 446-453. doi: 10.1136/ annrheumdis-2018-213678

Okada, Y., Wu, D., Trynka, G., Raj, T., Terao, C., Ikari, K., et al. (2014). Genetics of rheumatoid arthritis contributes to biology and drug discovery. Nature 506, 376-381. doi: 10.1038/nature12873

Park, M. J., Moon, S. J., Lee, E. J., Kim, E. K., Baek, J. A., Kim, S. Y., et al. (2019). Daurinol attenuates autoimmune arthritis via stabilization of Nrp1PTEN-Foxp3 signaling in regulatory T cells. Front. Immunol. 10:1526. doi: 10.3389/fimmu.2019.01526

Park, S. H., Kim, S. K., Choe, J. Y., Moon, Y., An, S., Park, M. J., et al. (2013). Hypermethylation of EBF3 and IRX1 genes in synovial fibroblasts of patients with rheumatoid arthritis. Mol. Cells 35, 298-304. doi: 10.1007/s10059-0132302-0

Plant, D., Webster, A., Nair, N., Oliver, J., Smith, S. L., Eyre, S., et al. (2016). Differential methylation as a biomarker of response to etanercept in patients with rheumatoid arthritis. Arthritis Rheumatol. 68, 1353-1360. doi: 10.1002/art. 39590

Qiu, H., Wu, H. J., Chan, V., Lau, C. S., and Lu, Q. J. (2017). Transcriptional and epigenetic regulation of follicular T-helper cells and their role in autoimmunity. Autoimmunity 50, 71-81. doi: 10.1080/08916934.2017.128 4821

Rhead, B., Holingue, C., Cole, M., Shao, X., Quach, H. L., Quach, D., et al. (2017). Rheumatoid arthritis naive $\mathrm{T}$ cells share hypermethylation sites with synoviocytes. Arthritis Rheumatol. 69, 550-559. doi: 10.1002/art. 39952

Rossetti, M., Spreafico, R., Saidin, S., Chua, C., Moshref, M., Leong, J. Y., et al. (2015). Ex vivo-expanded but not in vitro-induced human regulatory $\mathrm{T}$ cells are candidates for cell therapy in autoimmune diseases thanks to stable demethylation of the FOXP3 regulatory $\mathrm{T}$ cell-specific demethylated region. J. Immunol. 194, 113-124. doi: 10.4049/jimmunol.140 1145

Sekine, Y., Tsuji, S., Ikeda, O., Sato, N., Aoki, N., Aoyama, K., et al. (2006). Regulation of STAT3-mediated signaling by LMW-DSP2. Oncogene 25, 58015806. doi: 10.1038/sj.onc. 1209578

Shu, K., Kuang, N., Zhang, Z., Hu, Z., Zhang, Y., Fu, Y., et al. (2014). Therapeutic effect of daphnetin on the autoimmune arthritis through demethylation of proapoptotic genes in synovial cells. J. Transl. Med. 12:287. doi: 10.1186/ s12967-014-0287-x

Svendsen, A. J., Gervin, K., Lyle, R., Christiansen, L., Kyvik, K., Junker, P., et al. (2016). Differentially methylated DNA regions in monozygotic twin pairs discordant for rheumatoid arthritis: an epigenome-wide study. Front. Immunol. 7:510. doi: 10.3389/fimmu.2016.00510

Tabares, P., Berr, S., Langenhorst, D., Sawitzki, B., Ten Berge, I., Tony, H. P., et al. (2018). Short-term cytokine stimulation reveals regulatory T cells with downregulated Foxp3 expression in human peripheral blood. Eur. J. Immunol. 48, 366-379. doi: 10.1002/eji.201747244

Tang, C., Li, Y., Lin, X., Ye, J., Li, W., He, Z., et al. (2014). Prolactin increases tumor necrosis factor alpha expression in peripheral CD14 monocytes of patients with rheumatoid arthritis. Cell. Immunol. 290, 164-168. doi: 10.1016/j.cellimm.2014. 06.005

Tang, G., Pan, H., Xu, L., Feng, R., Jiang, Y., Kong, F., et al. (2018). A comparison of co-methylation relationships between rheumatoid arthritis and Parkinson's disease. Front. Neurosci. 12:1001. doi: 10.3389/fnins.2018. 01001

Toth, D. M., Ocsko, T., Balog, A., Markovics, A., Mikecz, K., Kovacs, L., et al. (2019). Amelioration of autoimmune arthritis in mice treated with the DNA methyltransferase inhibitor 5'-Azacytidine. Arthritis Rheumatol. 71, 1265-1275. doi: 10.1002/art.40877

Tseng, C. C., Lin, Y. Z., Lin, C. H., Li, R. N., Tsai, W. C., Ou, T. T., et al. (2019a). Genetic and epigenetic alteration of the programmed cell death 1 in rheumatoid arthritis. Eur. J. Clin. Investig. 49:e13094. doi: 10.1111/eci.13094

Tseng, C. C., Lin, Y. Z., Lin, C. H., Li, R. N., Yen, C. Y., Chan, H. C., et al. (2019b). Next-generation sequencing profiles of the methylome and transcriptome in peripheral blood mononuclear cells of rheumatoid arthritis. J. Clin. Med. 8:1284. doi: $10.3390 / \mathrm{jcm} 8091284$

van Steenbergen, H. W., Luijk, R., Shoemaker, R., Heijmans, B. T., Huizinga, T. W., and van der Helm-van Mil, A. H. (2014). Differential methylation 
within the major histocompatibility complex region in rheumatoid arthritis: a replication study. Rheumatology 53, 2317-2318. doi: 10.1093/rheumatology/ keu380

Wang, J., Li, J., Gu, J., Yu, J., Guo, S., Zhu, Y., et al. (2015). Abnormal methylation status of FBXW10 and SMPD3, and associations with clinical characteristics in clear cell renal cell carcinoma. Oncol. Lett. 10, 3073-3080. doi: 10.3892/ol.2015. 3707

Wang, X., Lei, D., Ding, J., Liu, S., Tao, L., Zhang, F., et al. (2018). A DNAmethylated sight on autoimmune inflammation network across RA, pSS, and SLE. J. Immunol. Res. 2018:4390789. doi: 10.1155/2018/4390789

Wu, W., Qin, M., Jia, W., Huang, Z., Li, Z., Yang, D., et al. (2019). Cystathionine-gamma-lyase ameliorates the histone demethylase JMJD3mediated autoimmune response in rheumatoid arthritis. Cell. Mol. Immunol. 16, 694-705. doi: 10.1038/s41423-018-0037-8
Yu, H., Ding, C., Dai, S., Sun, J., Wang, S., and Zhang, Z. (2019). Long noncoding RNA FER1L4 regulates rheumatoid arthritis via targeting NLRC5. Clin. Exp. Rheumatol. [Epub ahead of print].

Conflict of Interest: The authors declare that the research was conducted in the absence of any commercial or financial relationships that could be construed as a potential conflict of interest.

Copyright $\odot 2020 \mathrm{Guo}, \mathrm{Xu}$, Chang, Zhang, Jin and He. This is an open-access article distributed under the terms of the Creative Commons Attribution License (CC BY). The use, distribution or reproduction in other forums is permitted, provided the original author(s) and the copyright owner(s) are credited and that the original publication in this journal is cited, in accordance with accepted academic practice. No use, distribution or reproduction is permitted which does not comply with these terms. 